\title{
Dark soliton dynamics in spatially inhomogeneous media: Application to Bose-Einstein condensates
}

\author{
G. Theocharis ${ }^{\mathrm{a}}$, D.J. Frantzeskakis ${ }^{\mathrm{a}}$, P.G. Kevrekidis ${ }^{\mathrm{b}}$, \\ R. Carretero-González ${ }^{\mathrm{c}, *}$, B.A. Malomed ${ }^{\mathrm{d}}$ \\ ${ }^{a}$ Department of Physics, University of Athens, Panepistimiopolis, Zografos, Athens 15784, Greece \\ ${ }^{\mathrm{b}}$ Department of Mathematics and Statistics, University of Massachusetts, Amherst, MA 01003-4515, USA \\ ${ }^{c}$ Nonlinear Dynamical Systems Group, Department of Mathematics and Statistics, San Diego State University, \\ San Diego, CA 92182-7720, USA \\ d Department of Interdisciplinary Studies, Faculty of Engineering, Tel Aviv University, Tel Aviv 69978, Israel \\ Available online 6 July 2005
}

\begin{abstract}
We study the dynamics of dark solitons in spatially inhomogeneous media with applications to cigar-shaped Bose-Einstein condensates trapped in a harmonic magnetic potential and a periodic potential representing an optical lattice. We distinguish and systematically investigate the cases with the optical lattice period being smaller, larger, or comparable to the width of the dark soliton. Analytical results, based on perturbation techniques, for the motion of the dark soliton are obtained and compared to direct numerical simulations. Radiation effects are also considered. Finally, we demonstrate that a moving optical lattice may capture and drag a dark soliton.
\end{abstract}

(C) 2005 IMACS. Published by Elsevier B.V. All rights reserved.

Keywords: Soliton; Matter waves; Optical lattice; Bose-Einstein condensation

\section{Introduction}

Dark solitons (DSs), the most fundamental nonlinear excitations of the nonlinear Schrödinger (NLS) equation with a defocusing nonlinearity, have been widely studied in many branches of Physics.

\footnotetext{
${ }^{*}$ Corresponding author. Tel.: +1 619594 7252; fax: +1 6195942029.

E-mail address: carreter@math.sdsu.edu (R. Carretero-González).

URL: http://nlds.sdsu.edu/.
} 
Importantly, apart from the relevant theoretical work, there exist many experimental results, including the observation of optical dark solitons, either as temporal pulses propagating in optical fibers [1], or as spatial structures in bulk media and waveguides [2] (see also [3] for a review), the excitation of a non-propagating kink in a parametrically driven shallow liquid [4], dark-soliton standing waves in a discrete mechanical system [5], high-frequency dark solitons in thin magnetic films [6], etc.

Additionally, since the experimental realization of Bose-Einstein condensates (BECs) [7], there has been a considerable interest in DSs, which have been observed in a series of experiments [8]. As BECs are confined in magnetic traps, many theoretical studies have been devoted to the dynamics of DSs in the presence of external potentials $[9,10]$. In particular, it has been found that in elongated harmonic traps a DS oscillates with frequency $\Omega / \sqrt{2}$, where $\Omega$ is the axial trap frequency. Thermal [11] and dynamical [12] instabilities, mainly referring to rectilinear DSs, have been investigated. On the other hand, generalizations of the rectilinear solitons, such as ring-shaped DSs, have recently been proposed [13]. Systematic studies of the sound emitted by the DS due to inhomogeneities, as well as soliton-sound interactions have also been performed [14]. Furthermore, it has been shown that another dissipative effect, the so-called quantum depletion of DSs [15], reduces the dark soliton lifetime as atoms fill up the dark soliton notch.

Apart from the above, theoretical and experimental BEC studies have been performed in the case of a periodic external potential, the so-called optical lattice (OL) trap created by interference patterns from multiple laser beams [16-21]. The experimental control over the OL has led to the realization of numerous interesting phenomena including Bloch oscillations [18,22], Landau-Zener tunneling [16] (in the additional presence of a linear external potential), and classical [23] and quantum [21] superfluidinsulator transitions. With respect to the above, the dissipative dynamics of the DSs (including detailed quantitative measurements of the sound emitted by the soliton) in a quasi one-dimensional (1D) BEC confined through a harmonic trap and an OL, as well as the structure and the mobility properties of DSs in single and double-periodic OLs have recently been considered in [24,25], respectively. On the other hand, the stability of DSs in the combined harmonic trapping and OL potential, in both the discrete and the continuum mean-field model framework, has recently been studied [26].

From the more general point of view of the theory of nonlinear waves and solitons, the aforementioned studies can be considered as complementary to the ones related to the soliton dynamics in spatially inhomogeneous, as well as in disordered media, a topic of practical relevance in diverse fields (see, e.g., [27]). Especially, as far as the bright solitons of the nonlinear Schrödinger (NLS) equation are concerned, it has been shown that their dynamics is strongly affected by the competition of the lengthscales characterizing the solitons and the spatially inhomogeneous perturbations [28]. In this respect, it is relevant to note the following: first, the dark soliton dynamics in quasi 1D BECs can also be described in the framework of an NLS equation, the so-called Gross-Pitaevskii (GP) equation [7]. Second, in the case where the BEC is confined by a harmonic trap and an OL at the same time, there exist three different length scales, i.e., one referring to the DS, one to the harmonic trap and one to the OL. It is therefore expected that different dynamical regimes for the DS are possible, while interesting effects resulting from a length-scale competition may arise.

The scope of this work is to contribute in this direction, systematically studying the dynamics of DSs in spatially inhomogeneous media, with the inhomogeneity induced by the presence of external potentials, e.g., the confining harmonic trapping and the periodic OL, both appearing naturally in the BEC context. Particularly, we aim to identify and analyze the different regimes of the DS dynamics, relevant to the entire range of scales of the lattice periodicity, as compared to the scales of the DS and the confining 
potential. We consider the following normalized defocusing NLS (or GP) equation:

$$
i u_{t}=-\frac{1}{2} u_{x x}+|u|^{2} u+V(x) u .
$$

which can be used as a mean-field model describing the dynamics of a cigar-shaped BEC with repulsive interatomic interactions $[7,29,30]$, trapped in the potential:

$$
V(x)=\frac{1}{2} \Omega^{2} x^{2}+V_{0} \cos ^{2}(k x),
$$

where the two terms represent the magnetic trap (MT) and the OL, respectively. In this context, $u(x, t)$ in Eq. (1) is the macroscopic wave function of the BEC, $t$ and $x$ are measured, respectively, in units of $\omega_{\perp}^{-1}$ and the transverse harmonic-oscillator length, $a_{\perp}=\left(\hbar / m \omega_{\perp}\right)^{1 / 2}$. Accordingly, the parameter $\Omega \equiv \omega_{x} / \omega_{\perp}$, where $\omega_{x}$ and $\omega_{\perp}$ are the confining frequencies in the axial and transverse directions, determines the strength of the MT. The strength of the OL $V_{0}$ is measured in units of the recoil energy $E_{r} \equiv h^{2} / 2 m \lambda_{\text {laser }}^{2}$, where $\lambda_{\text {laser }}$ is the wavelength of the laser beams producing the optical trap. Finally, $k$ is the wavenumber of the OL that can be experimentally controlled by varying the angle $\theta$ between the counter-propagating lasers producing the interference pattern of wavelength $\lambda=2 \pi / k=\lambda_{\text {laser }} \sin (\theta / 2) / 2$ [31].

In the absence of the external (MT and OL) potentials, i.e., for an homogeneous BEC, the defocusing NLS equation (1) possesses an exact solution for the DS. Particularly, on a homogeneous background density $u_{0}^{2}$, the DS has the form [32]:

$$
u(x, t)=u_{0}(\cos \varphi \tanh \zeta+\mathrm{i} \sin \varphi) \exp (-\mathrm{i} \mu t) .
$$

Here $\mu \equiv u_{0}^{2}$ is the one-dimensional chemical potential, determined by the number of atoms of the condensate, $\varphi$ is the phase shift $(|\varphi|<\pi / 2)$ across the DS, $\zeta \equiv u_{0}(\cos \varphi)\left[x-u_{0}(\sin \varphi) t\right]$, while the amplitude and velocity of the DS are given by $u_{0} \cos \varphi$ and $u_{0} \sin \varphi$, respectively; the limit case $\varphi=0$ corresponds to a quiescent DS, $u=u_{0} \tanh \left(u_{0} x\right) \exp (-\mathrm{i} \mu t)$. In the presence of the MT and OL potentials, the background density supporting the DS is nonuniform, and can be well approximated by the so-called Thomas-Fermi (TF) wave function [7]:

$$
u_{\mathrm{TF}}=\sqrt{\max \{0, \mu-V(x)\}} .
$$

Note that $\mu$ may be scaled out, therefore we hereafter set $\mu \equiv 1$.

To distinguish between different physically relevant cases, it is necessary to identify the length scales involved in the problem. First, the MT strength $\Omega$ in Eq. (2) sets the corresponding length scale $\Omega^{-1}$, which we assume to be much larger than the DS width $l_{\mathrm{DS}} \equiv(\cos \varphi)^{-1}$ (recall we have set $u_{0}^{2}=\mu \equiv 1$ ), otherwise (if MT is tight, rather than loose) the DS may not exist [26]. Note that, unless the DS is very shallow (i.e., $\cos \varphi \ll 1$ ), which is not a case of interest, $l_{\mathrm{DS}}$ is on the order of magnitude of the healing length $\xi$, that, in the framework of Eq. (1) with $\mu \equiv 1$, is $\xi=\sqrt{2}$; this actually means that $\Omega \ll 1$. On the other hand, as concerns the OL potential, both its strength $V_{0}$ and wavelength $\lambda$ may be, generally speaking, arbitrary, hence the ratio of the DS width to $\lambda$ may take different values. Therefore, it is relevant to single out three cases, namely, a long-period OL, with $\lambda \gg l_{\mathrm{DS}} \sim \xi$, a short-period OL, with $\lambda \ll \xi$, and an intermediate case, with $\lambda \sim \xi$.

First, the long-period OL case will be studied below. In this case the full potential (including the MT and OL terms) varies slowly on the DS scale and the soliton dynamics can be treated analytically by means of the adiabatic perturbation theory for DSs [33] (see also Refs. [10,13] for applications of the perturbation theory in the BEC context). This way, we will demonstrate analytically (and confirm by direct numerical 
simulations) that the DS oscillates on top of the TF cloud with a frequency and amplitude modified by the presence of the OL. We will also investigate the role of the strength $V_{0}$ of the OL potential. For small values of $V_{0}$, the soliton evolves almost adiabatically, as the inhomogeneity-induced radiation of the DS in the form of sound waves is practically negligible, and its motion can be described analytically; nevertheless, as $V_{0}$ takes larger values, the adiabatic perturbation theory fails, since the sound emission becomes gradually stronger. Thus, due to this recoil effect, the DS becomes shallower and more mobile, and, as a result, the DS performs oscillations of small-frequency and large amplitude.

Next, we will consider the case of a short-period OL $(\lambda \ll \xi)$. In this case, we apply a multi-scale perturbation theory [34]. It will be shown that, quite naturally, the influence of the OL is averaged out, i.e., the DS obeys an effective NLS (GP) equation with a potential solely composed by an effective MT with a modified strength.

Finally, we will consider the intermediate case, when the OL period is of the order of the healing length $(\lambda \sim \xi)$. In this case, we will show that, as long as the initial position of the DS is sufficiently close to the bottom of a local well of the OL potential, and the OL is relatively weak $\left[V_{0}=O(1)\right]$, the DS emits radiation at a small rate. Thus, the soliton remains trapped in the well for a long time, but will eventually escape, and start performing large-amplitude low-frequency oscillations in the condensate.

Using the fact that there are cases where the soliton remains trapped for relatively long times, we also propose a possibility of "targeted transfer" of the DS by an adiabatically moving OL, which is properly phase-modulated. This result demonstrates that DSs can be manipulated by time-dependent OLs, which may find applications in matter-wave devices. The paper is organized as follows. In Section 2 we examine the case of a long-period OL, in Section 3 we study the short-period one, and the intermediate case is considered in Section 4. In the same section we demonstrate the possibility to capture and drag the DS by a time-dependent OL. Finally, Section 5 contains the conclusions.

\section{The long-period optical lattice}

We first consider the case when the OL period is much larger than the DS width. Then, assuming that the strength of the OL potential is small enough, the DS dynamics can be treated perturbatively. To this end, we seek a DS solution of Eq. (1) on top of a TF cloud. As the MT strength is always taken to be small, we may define a region (close to the center of the trap) where the trap potential is much smaller than the chemical potential $\mu$ (recall we have set $\mu \equiv 1$ ). Thus, approximating the TF wave function (4) by the expansion $u_{\mathrm{TF}}^{2} \approx \max \{0,1-V(x) / 2\}$, we seek for a solution to the GP equation of the form $u=u_{\mathrm{TF}}(x) v(x, t)$, where the complex field $v(x, t)$ describes the DS. Then, we follow the procedure described in Ref. [10] (see also Ref. [13]) to derive the following effective perturbed NLS equation from Eq. (1):

$$
i \frac{\partial v}{\partial t}+\frac{1}{2} \frac{\partial^{2} v}{\partial x^{2}}-\left(|v|^{2}-1\right) v=P(v),
$$

where the effective perturbation is

$$
P(v) \equiv\left(1-|v|^{2}\right) v V+\frac{1}{2} \frac{\mathrm{d} V}{\mathrm{~d} x} \frac{\partial v}{\partial x}
$$


$V$ being the same potential (including the MT and OL terms) as in Eq. (2). In the general case, all the terms in the perturbation (6) are of the same order of magnitude (smallness).

Next, assuming that the perturbation $P$ is small indeed, we apply the perturbation theory for DSs, as developed in Ref. [33], to Eq. (5). This way, we start with the unperturbed DS, and seek for a solution to Eq. (5) of the form:

$$
v(r, t)=\cos \varphi(t) \tanh \zeta+\mathrm{i} \sin \varphi(t),
$$

where $\zeta=\cos \varphi(t)\left(x-x_{0}(t)\right)$ [cf. Eq. (3)], and $\varphi(t)$ and $x_{0}(t)$ are the slowly varying phase and position of the DS. Then, it is straightforward to derive the equation of motion for the center of the initially quiescent $(\varphi(0)=0) \mathrm{DS}$ :

$$
\frac{\mathrm{d}^{2} x_{0}}{\mathrm{~d} t^{2}}=-\frac{\mathrm{d} V_{\text {eff }}}{\mathrm{d} x_{0}}
$$

with the effective potential:

$$
V_{\mathrm{eff}}(x)=\frac{\Omega^{2}}{4} x^{2}+\frac{V_{0}}{4}\left[1-\left(\frac{\pi^{2}}{3}-2\right) \frac{k^{2}}{6}\right] \cos (2 k x) .
$$

It is readily observed that in the absence of the OL, Eq. (8) recovers the result predicted in Refs. $[9,13,10]$, i.e., the DS oscillates in the harmonic trap with the frequency $\omega=\Omega / \sqrt{2}$. The combination of the MT and OL gives rise to the additional periodic term in the effective potential (9). Here it is relevant to note that the sign of the coefficient following $V_{0}$ in Eq. (9) is always positive as (by assumption) $k^{2} \ll 1$.

For a DS placed near the bottom of the magnetic trap $\left(x_{0}=0\right)$, Eqs. (8) and (9) show that the effective oscillation frequency is

$$
\omega_{\mathrm{eff}} \approx \sqrt{\frac{1}{2} \Omega^{2}-V_{0} k^{2}}
$$

provided that $k<\Omega / \sqrt{2 V_{0}}$. The analytical result (10) was verified against direct numerical simulations of the GP equation (1). The numerical simulations presented in this work were carried out by integrating Equation (1) using a pseudo-spectral method [35] with, typically, $2^{9}$ to $2^{10}$ mesh points and a time step of $\mathrm{d} t=0.01$. Fig. 1 demonstrates that the trajectory of the DS center, as predicted by Eq. (8) (dotted line) is almost identical to the respective one found from direct integration of Eq. (1) (solid line), while the motion of the soliton in the absence of the OL is clearly characterized by a larger frequency (dashed line). Assuming that the strengths of the magnetic trap and OL are $\Omega=0.075$ and $V_{0}=0.07$, and $k=0.1$, the DS, initially placed at $x_{0}(0)=-1.5$, performs oscillations in the presence (absence) of the OL with the frequency $0.046(0.054)$, while the corresponding analytical predictions are $\omega_{\text {eff }}=0.04596$ $(\omega=0.05303)$. Thus, the numerically found oscillation frequencies are in very good agreement with the analytically predicted values.

Here, it should be noted that the soliton trajectories are shown in Fig. 1 up to $t=500$, which, according to values of the physical parameters used in relevant experiments with DSs in BECs [8], corresponds to $\approx 100 \mathrm{~ms}$. Although such a long-time description is quite relevant from the point of view of the theory of nonlinear waves, in the context of BECs important dissipative effects, such as the interaction of the configuration with the thermal cloud [11] or/and the quantum depletion of the DS [15], may significantly reduce the soliton lifetime. 


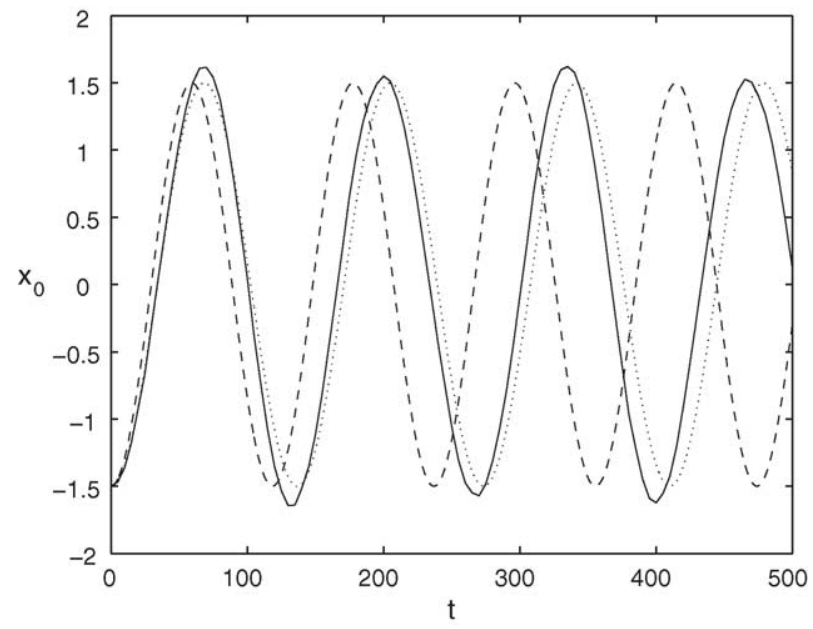

Fig. 1. Motion of the center of a dark soliton, initially placed at $x_{0}(0)=-1.5$, in the presence (solid line and dotted lines) or absence (dashed line) of the optical lattice. The analytical prediction (dotted line) is in very good agreement with the numerical result (solid line), while the oscillation frequency of the dark soliton in the absence of the optical lattice is clearly larger (dashed line). The parameters are $\Omega=0.075, V_{0}=0.07$ and $k=0.1$.

We have also considered the case where the initial position of the DS center is not close to the bottom of the potential well. In this case, the oscillation frequency of the dark soliton center can be found, either integrating the underlying GP equation (1), or the quasi-particle equation of motion (8). We have found that the agreement between both results (for the oscillations of the DS center) is again quite good. As an example, in Fig. 2(a) we show the motion of the DS center, initially placed at $x_{0}(0)=-10 \pi+20$, with OL parameters $V_{0}=0.05$ and $k=0.05$ (the MT strength is $\Omega=0.005$ ). The trajectory found by the integration of the GP equation (solid line) almost coincides with the one found from the solution of the quasi-particle's equation (8) (dotted line). Also, the DS remains almost undistorted up to large times, see the snapshot for $t=1000$ in Fig. 2(b); this result is somewhat expected, due to the fact that in this case we deal with weak traps and OL strengths, so the deformation of the soliton due to the sound emission demonstrated in [24] is a negligible effect.

As long as the OL strength $V_{0}$ remains small enough, i.e., for $V_{0}<0.1$, the DS evolves almost adiabatically, as the radiation (sound in the form of linear waves) emitted by the soliton due to the system's inhomogeneity is practically negligible. In this case, the adiabatic perturbation theory outlined above describes the soliton motion quite accurately. However, for larger values of $V_{0}$, the inhomogeneity induced by the OL becomes stronger and the sound emission becomes important. In such a case, the DS becomes shallower and gains velocity due to the recoil effect; this, in turn, makes the amplitude and frequency of the oscillations, respectively, larger and smaller than those predicted by the perturbation theory. This situation is demonstrated in Fig. 3, where the evolution of the DS is shown, with the same initial position and MT potential as in Fig. 2, but for a significantly stronger OL, with $V_{0}=0.5$. As seen in Fig. 3(a), where a detail of the TF configuration with the soliton is shown at $t=25$, the emission of radiation starts immediately and is apparent even at the initial stages of the evolution. As follows from Fig. 3(b), although there is good initial agreement (for the motion of the soliton center) between the analytical prediction based on the perturbation theory (dashed line) and numerical solution of the GP equation (solid line), as time advances the soliton oscillations are characterized by a larger amplitude and a smaller frequency. 

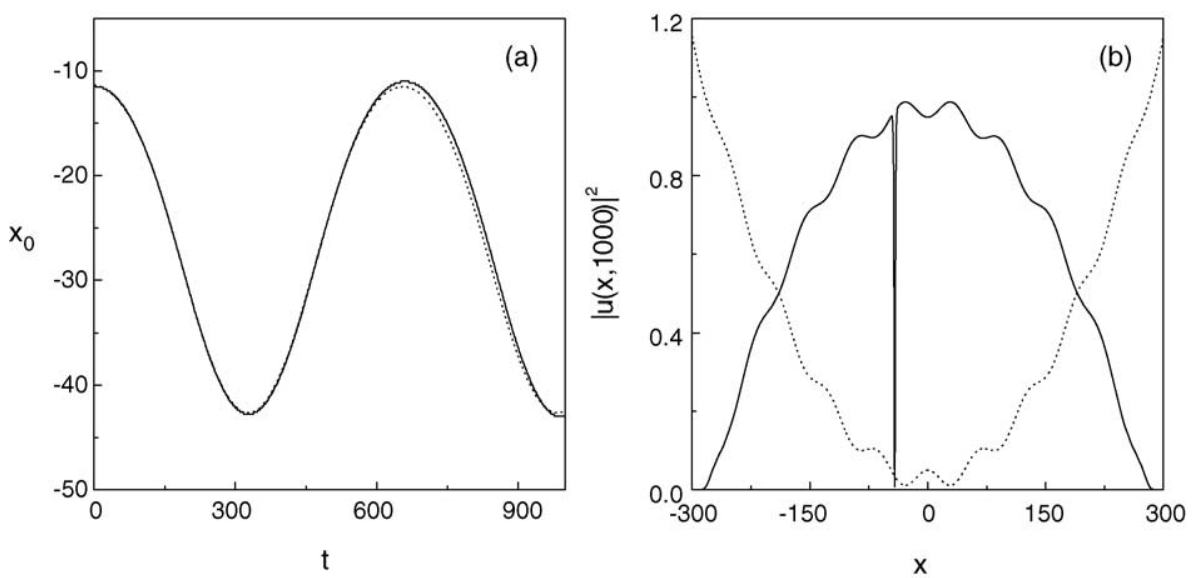

Fig. 2. (a) Motion of the center of a dark soliton, initially placed at $x_{0}(0)=-10 \pi+20$, in the presence of the magnetic trap with $\Omega=0.005$ and optical lattice with $V_{0}=0.05$ and $k=0.05$. The almost identical solid and dotted lines correspond to the solution of the GP equation (1) and the quasi-particle equation of motion (9), respectively. (b) Snapshot of the density $|u(x)|^{2}$ (solid line) at $t=1000$. The dotted line shows the full (MT and OL) external potential.

This case cannot be described analytically, as the adiabatic perturbation theory does not apply at large values of the OL strength. Note that, at $t=1000$, the soliton is still oscillating in the potential well of the OL where it was initially placed. For even larger times (not shown here), it is natural to expect that it will escape from the well and will further increase (decrease) the amplitude (frequency) of the oscillation (see also a discussion in the next sections, as well as $[14,24]$ for a detailed description of the effect of radiation).
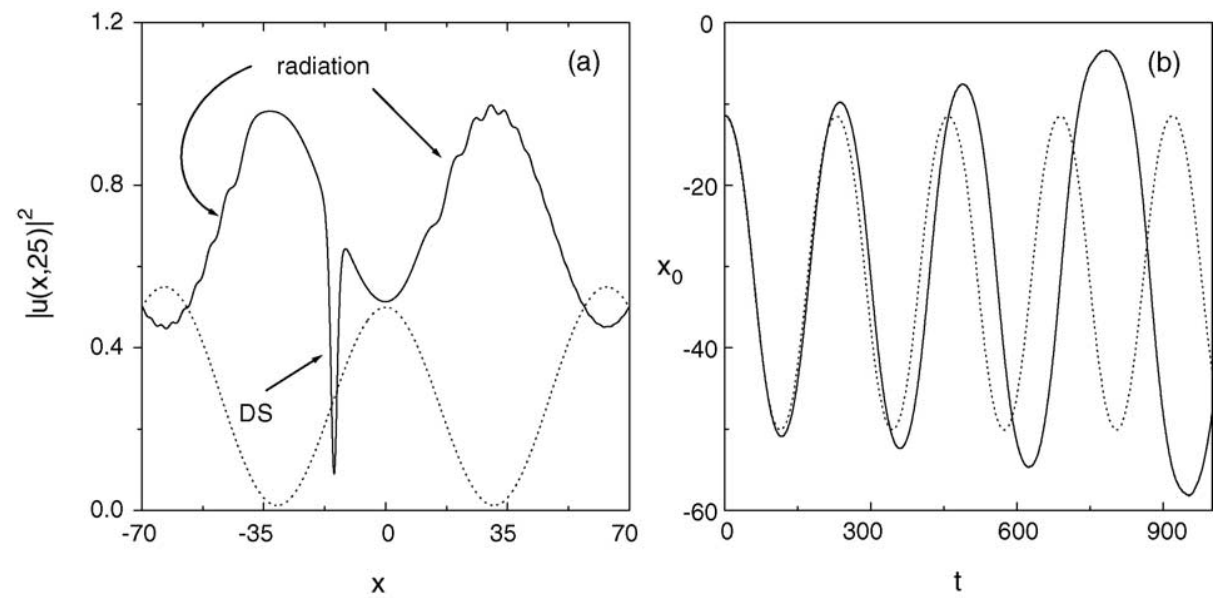

Fig. 3. (a) A detail of the atomic density of the condensate cloud (solid line), incorporating a dark soliton, at $t=25$ (10 ms), for the same initial configuration as in Fig. 2, but with the optical lattice strength $V_{0}=0.5$; the emission of radiation is apparent. The dotted line shows the full (MT and OL) external potential. (b) Motion of the DS center, with the solid and dotted lines corresponding to the solution of the GP equation (1) and the equation of motion (8), respectively. It is seen that the emission of radiation causes the soliton to perform larger-amplitude, low-frequency oscillations. 


\section{The short-period optical lattice}

We now proceed to the case when the OL period is much smaller than the width of the dark soliton (i.e., $\lambda \ll \xi$, or $k \gg 1$ ). In this case, $k^{-1}$ can be naturally considered as a perturbation parameter. The MT strength $\Omega$ is assumed to be on the order of $k^{-2}$ (which corresponds to the most interesting situation), while the OL strength $V_{0}$ is $O(1)$, i.e., it is not small.

As the length scales of the MT and OL potentials are very different, the wave function $u(x, t)$ is sought for as a sum of slowly and rapidly varying parts, $U(x, t)$ and $w(x, t)$, respectively:

$$
u(x, t)=\exp \left[-\mathrm{i}\left(\frac{V_{0}}{2}\right) t\right][U(x, t)+w(x, t)] .
$$

Note that the multiplier $\exp \left[-\mathrm{i}\left(V_{0} / 2\right) t\right]$ in Eq. (11) is introduced so as to produce a term $\left(V_{0} / 2\right) u$ in the GP equation (1), which is cancelled out by the corresponding "dc part" of the OL potential term. In order to derive an effective equation for the slowly varying part $U$ of the wave function, we apply the asymptotic procedure described in Ref. [34]. In particular, we represent the rapidly varying part of the wave function as a Fourier series:

$$
w(x, t)=\sum_{n=1}^{\infty}\left[A_{n} \cos (2 n k x)+B_{n} \sin (2 n k x)\right],
$$

where the functions $A_{n}$ and $B_{n}$ are assumed to be slowly varying. Upon substituting Eqs. (11) and (12) into Eq. (1) and equating coefficients in front of the harmonics, we arrive at a system of coupled equations for $A_{n}$ and $B_{n}$. To solve these equations, we assume asymptotic expansions in powers of $k^{-1}$ :

$$
A_{n}=\sum_{j=1}^{\infty} \frac{a_{n}^{j}}{(2 k)^{2 n}}, \quad B_{n}=\sum_{j=1}^{\infty} \frac{b_{n}^{j}}{(2 k)^{2 n+1}} .
$$

Eventually, in the leading-order approximation, we derive an evolution equation for the slowly varying component $U$ :

$$
i p U_{t}+\frac{1}{2} q U_{x x}-r|U|^{2} U=-\frac{1}{32} \delta^{2} U+\frac{1}{2} \Omega^{2} x^{2} U,
$$

where $\delta \equiv V_{0} / k$, and

$$
p=1+\frac{1}{64} \frac{\delta^{2}}{k^{2}}, \quad q=1-\frac{3}{128} \frac{\delta^{2}}{k^{2}}, \quad r=1-\frac{9}{128} \frac{\delta^{2}}{k^{2}} .
$$

Finally, upon rescaling the spatial variable and the wave function, with $X=(p / q)^{1 / 2} x$ and $U=$ $(p / r)^{1 / 2} \exp \left[\mathrm{i}\left(\delta^{2} / 32 p\right) t\right] \Phi$, Eq. (14) is cast in the form:

$$
i \Phi_{t}+\frac{1}{2} \Phi_{X X}-|\Phi|^{2} \Phi=\frac{1}{2}\left(\frac{q}{p^{2}}\right) \Omega^{2} X^{2} \Phi .
$$

Thus, the averaged dynamics of the DS in the presence of the rapidly varying OL potential is described by the renormalized GP equation (16). This suggests that the DS behaves as a "renormalized quasi-particle", which oscillates in the MT as per Eq. (8), but with an effective potential $V_{\text {eff }}\left(x_{0}\right)$ (recall that $x_{0}$ is the DS central coordinate) now given by $V_{\text {eff }}=(1 / 4) q p^{-2} \Omega^{2} x_{0}^{2}$. According to this analysis, the frequency of 

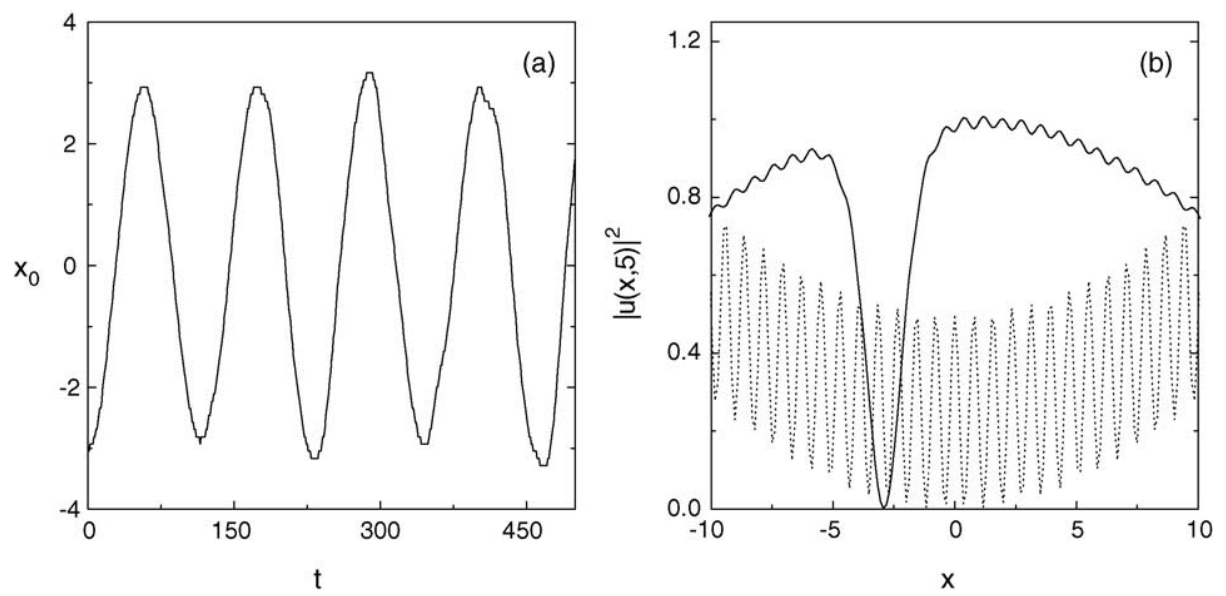

Fig. 4. (a) Motion of the center of the dark soliton, initially placed at $x_{0}(0)=-3$, in the presence of the magnetic trap with the strength $\Omega=0.075$ and the optical lattice with $V_{0}=0.5$ and $k=4$. The oscillation frequency is very close to the analytically predicted value (cf. Eq. (17)), $\Omega_{\text {eff }} \approx 0.053$. (b) The profile of the dark soliton at $t=5$ ( $2 \mathrm{~ms}$; solid line), and the corresponding external potential including the magnetic trap and optical lattice (dotted line).

oscillation $\Omega_{\text {eff }}$ is given by

$$
\Omega_{\mathrm{eff}} \approx \frac{\Omega}{\sqrt{2}}\left(1-\frac{7}{256} \frac{\delta^{2}}{k^{2}}\right) .
$$

It is important to note that the above result concerning the renormalization of the DS characteristics bears resemblance to the renormalization of the atomic mass of the condensate, i.e., to the existence of an effective mass induced by the OL potential, which has been reported in [36] (see also a relevant discussion in [24]).

Direct numerical integration of the GP equation has confirmed the above findings. In particular, assuming the MT strength $\Omega=0.075$ and the OL strength $V_{0}=0.5$ and wavenumber $k=4$, we have found that the DS initially placed at $x_{0}(0)=-3$ performs oscillations with a frequency approximately equal to 0.053 , corresponding to the value $\Omega / \sqrt{2}$, the higher-order correction in Eq. (17) being of $O\left(10^{-5}\right)$. The soliton, indeed, does not "feel" the presence of the short-scale OL potential, as is clearly seen from the motion of the center of the soliton in Fig. 4.

\section{Intermediate optical lattice period}

The particle-like description of the DS adopted in the previously considered cases may also be helpful when the period of the OL potential is of the same order of magnitude as the DS width, i.e., $\lambda \sim \xi$ or $k=O(1)$. In such a case, one may expect that if a stationary DS is initially placed in a well of the OL potential, it will remain trapped there for long times. This has been confirmed by direct simulations (see below), which have shown that the trapping time may be on order of $t \approx 750$ (300 ms). On the other hand, if the dark soliton is placed near a potential maximum rather than minimum, it stays there up to $t \approx 50$ (20 ms) only, and then moves away. This "quasi-trapping" of the DS around the minimum of the potential 

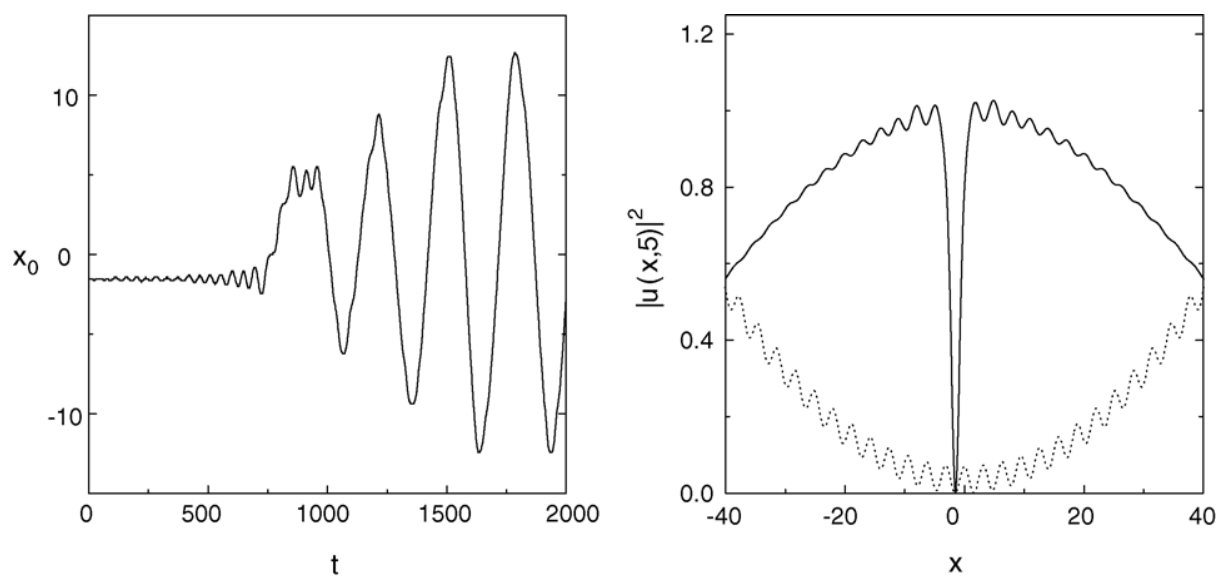

Fig. 5. Left panel: motion of the center of a dark soliton, initially placed at $x_{0}(0)=-1.5$ in the presence of the magnetic trap with $\Omega=0.025$ and optical lattice with $V_{0}=0.07$ and $k=1$. It is seen that up to $t \approx 770$ the soliton remains trapped in the well of the optical lattice potential which is the closest to the condensate's center; then, after emitting radiation, the dark soliton gets shallower and escapes, oscillating in a wider area of the condensate. The density of the dark soliton (solid line) and the full potential (dashed line) are also shown at $t=5$ (right panel).

energy is also expected to occur for small deviations of the initial soliton's position from the bottom of the potential well, but the trapping time will be shorter. In any case, the soliton eventually escapes from the quasi-trapped state, and moves on top of the TF state (which, in the case under consideration, is highly inhomogeneous). The escape is accounted for by radiative effects that in some cases lead to the destruction of the DS (relevant results concerning the stability of the DS in BECs confined in OLs have recently been reported in [26]).

In order to systematically investigate this regime, we have first performed numerical integration of the GP equation, with an initial condition $u(x, 0)=\tanh (x-1.5)$. This corresponds to a dark soliton initially placed near the bottom of the OL-potential well closest to the center of the condensate (the bottom of this well is located at $x=-\pi / 2$ ). The MT and OL parameters were taken to be $\Omega=0.025$, $V_{0}=0.07$ and $k=1$. The result is displayed in Fig. 5, where the motion of the soliton center and a snapshot of the soliton density at an initial stage of its evolution (at $t=5$ ) are shown. It is seen that up to $t \approx 80$ the DS remains almost completely stationary. Then, after emitting a small amount of radiation, it becomes shallower, thus gaining velocity and starting to oscillate inside the well of the OL potential. These small-amplitude oscillations last up to $t \approx 770$; after that, the soliton has gained the kinetic energy necessary to escape from the well and move to a neighboring one. There, it is again trapped due to a reabsorption of the radiation waves: this effect [14] causes the soliton to become deeper, which results in loss of its kinetic energy, and a subsequent stabilization of its motion. The soliton oscillates in that well from $t=825$ up to $t=980$. Eventually, further emission of radiation results again in the increase of the soliton's kinetic energy and the amplitude of oscillations increases. Note that at $t=2000$ the soliton position is $x \approx-3.5$, i.e., quite close to its initial location, while its amplitude is $\cos \varphi \approx 0.95$; thus, at that time, the soliton intensity defined as $\cos ^{2} \varphi$ [33], takes $90 \%$ of its initial value (recall that $\left.\cos ^{2} \varphi(0)=1\right)$.

For larger times $(t>1500)$, the oscillation frequency of the soliton seems to saturate at a value approximately equal to 0.021 , which is quite close to $\Omega_{\text {eff }} \approx 0.018$. The latter is the prediction of Eq. (17), which 

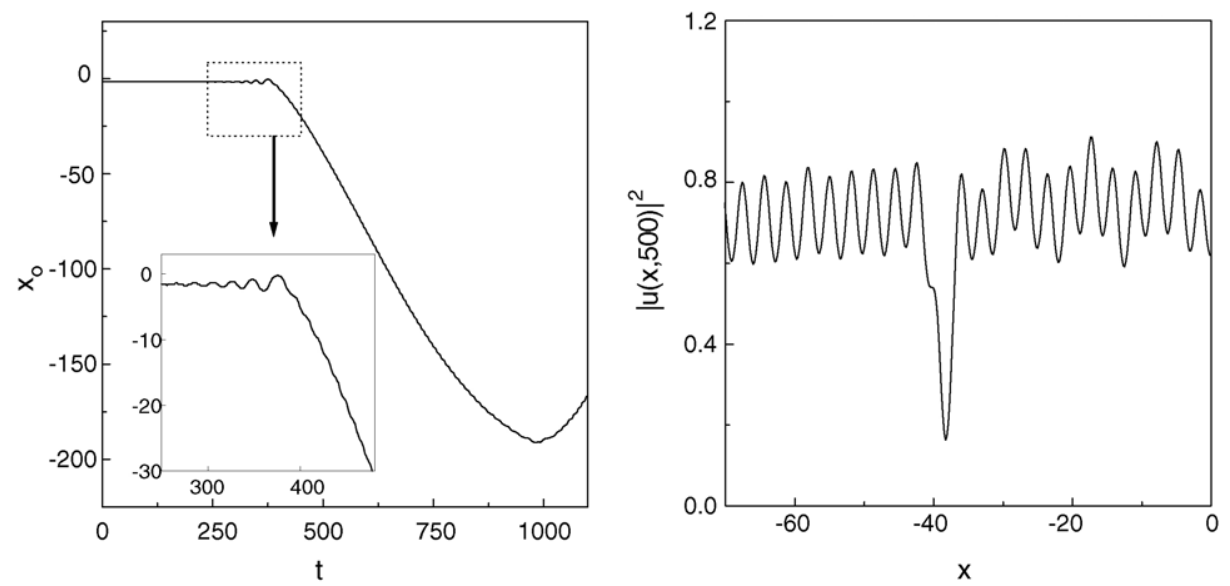

Fig. 6. Left panel: motion of the center of the dark soliton, initially placed at $x_{0}(0)=-\pi / 2$, in the presence of the magnetic trap with $\Omega=0.005$ and optical lattice with $V_{0}=0.5$ and $k=1$. The soliton remains almost immobile up to $t \approx 250$, and then it starts to oscillate inside the OL well and finally escapes with some acceleration, as shown in the inset, to reach the rim of the TF cloud. Right panel: a detail of the condensate density, incorporating the dark soliton, is shown at $t=500$; considerable distortion of the condensate and soliton is apparent.

is, of course, relevant to the case considered above in Section 3. However, in the case under consideration, although the initial amplitude (depth) and width of the dark soliton were approximately equal to 1 , at large times its amplitude has decreased (due to the emission of radiation), rendering its width larger. Thus, at large times we deal with a DS whose width is larger than the OL period, suggesting that the results obtained in the previous section may be relevant.

We have also examined the case when the initial position of the DS was at a bottom of a well of the OL potential, while the MT potential was weaker, in order to further clarify the interaction of the DS with the OL. Such a case is shown in Fig. 6, in which we have set the initial position of the DS at $x_{0}=-\pi / 2$, the MT strength $\Omega=0.005$, and an OL with $V_{0}=0.5$ and $k=1$.

It is observed that the soliton remains immobile up to $t \approx 250$, and then it starts to oscillate in the well with increasing amplitude, see the inset in Fig. 6 (left). Then, at $t \approx 380$ the soliton gains enough kinetic energy to escape from the well. Careful consideration demonstrates that the soliton escapes from the trap with some acceleration [this may be seen in the inset of Fig. 6 (left)], thus continuously losing its energy [37] (see also discussion in Ref. [14]). This energy loss renders the soliton shallower. As an example, we note that at $t=500$ the soliton's amplitude (depth) is approximately 0.77 , as seen in Fig. 6 (right), while, at the same time, the condensate itself is clearly distorted. The dark soliton continues its path to the rim of the TF cloud, where it gets reflected (this occurs at $t \approx 1000$ ). Later, at $t \approx 1100$, the soliton amplitude falls to approximately 0.5 , i.e., it becomes comparable to the strength of the OL, hence it can no longer be distinguished from the TF background. In fact, the DS has essentially completed its decay by that time.

In general, we have found that the emission of radiation is relatively strong (thus resulting in smaller soliton-trapping times) as long as the strength $V_{0}$ of the OL potential and/or the deviation of the initial soliton's position from the bottom of a lattice potential well are relatively large. We have also found that the increase of the wavenumber $k$ plays an important role in the manifestation of the radiation effects. As an example, in Fig. 7, we show the configuration at $t=5$, together with the motion of the DS initially 

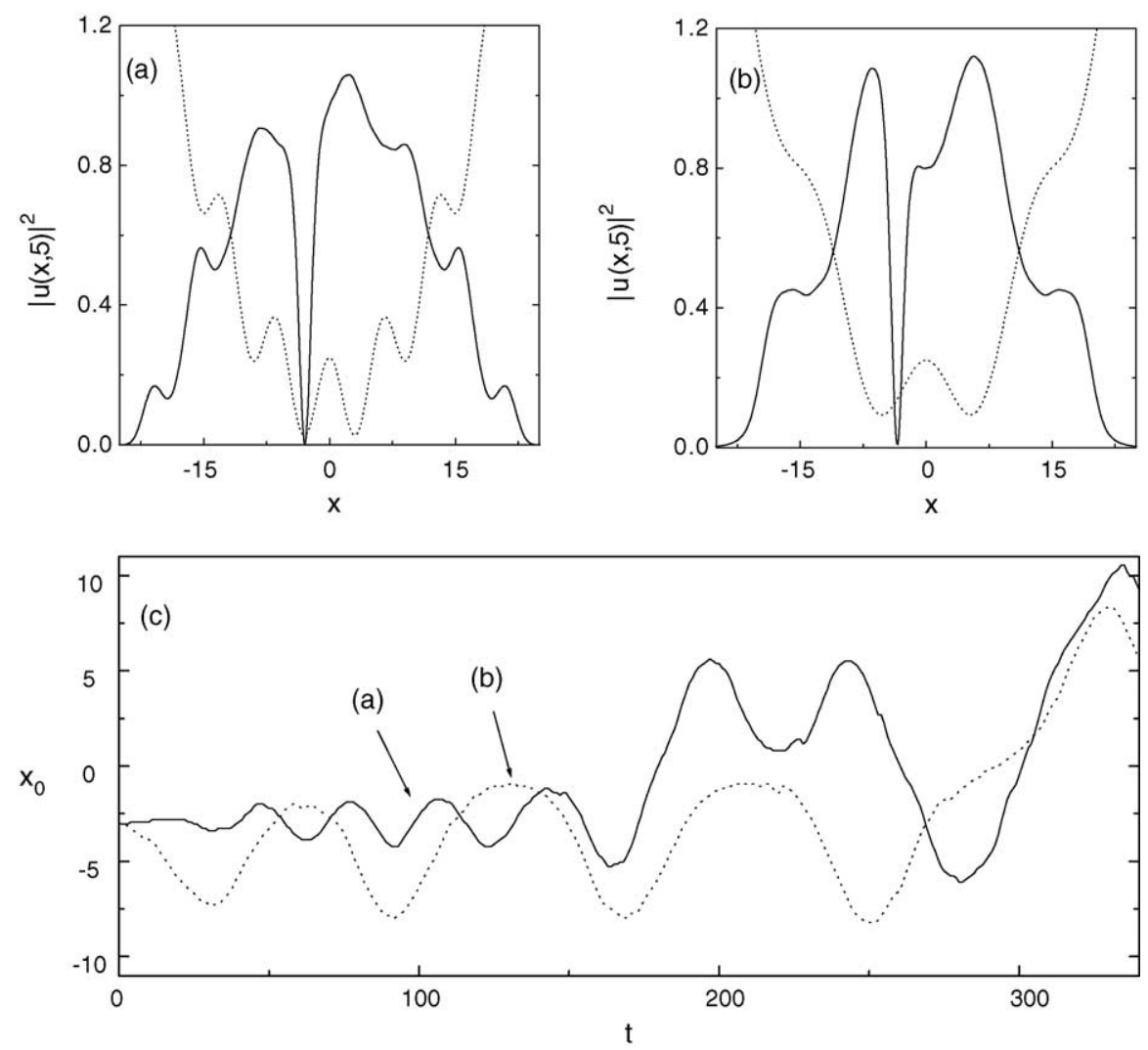

Fig. 7. ( $\mathrm{a}$ and $\mathrm{b}$ ) The density of the dark soliton at $t=5$ (solid line), initially placed at $x_{0}(0)=-3$; the potential is shown by the dashed line. The strengths of the magnetic trap and optical lattice potentials are $\Omega=0.075$ and $V_{0}=0.25$, respectively. The wavenumber of the optical lattice is (a) $k=0.5$ and (b) $k=0.25$. (c) Motion of the center of the dark soliton for the above-mentioned configurations (a) (solid line) and (b) (dotted line).

placed at $x_{0}(0)=-3$, for two different cases, namely, $k=0.5$ and $k=0.25$ (the MT and OL strengths are $\Omega=0.075$ and $V_{0}=0.25$ ). In the former case, the soliton is placed at the bottom of the well closest to the condensate's center. As a result, the behavior is quite similar to that observed in Fig. 5: initially, the soliton stays trapped in the well, but at $t \approx 175$ it escapes through the radiation-emission mechanism described above. Subsequently, it oscillates inside the next well (up to $t \approx 270$ ), then it escapes again and oscillates in a wider region inside the condensate. In the second case (with $k$ twice as small), the DS is not placed at the bottom of the well, therefore it starts to oscillate immediately. The oscillations last up to $t \approx 280$, and then the soliton gains sufficient kinetic energy to escape, thus switching to oscillations with a larger amplitude. These results show that, for increasing $k$, the transient trapping of the DS in the potential well ends earlier (despite the DS being initially located at the potential minimum).

The fact that we have identified cases when the soliton remains trapped for long times in a well of the optical lattice suggests the following possibility: consider a slowly moving OL potential which initially traps a DS. The slow motion of the OL can be induced by varying the phase difference $\Delta$ between the two laser beams that form the lattice, the velocity of the motion being $c=(\pi / k) \mathrm{d} \Delta / \mathrm{d} t[31]$. Then, a natural question is whether this steadily moving OL can drag the initially trapped DS and bring it to 


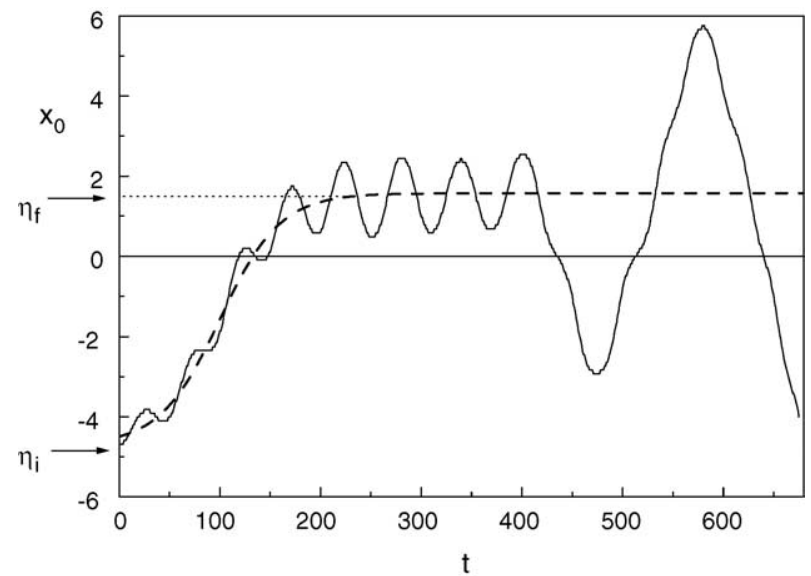

Fig. 8. Targeted transfer of a dark soliton from its initial position, $\eta_{\mathrm{i}}=-3 \pi / 2$, to the final one, $\eta_{\mathrm{f}}=\pi / 2$. The strength of the magnetic trap is $\Omega=0.025$, the optical lattice parameters are $V_{0}=0.07$ and $k=1$, and the transfer law is taken with $t_{0}=100$ and $T=60$ [see Eqs. (18) and (19)]. The soliton delivered by the OL to the pre-selected destination stays there, performing small oscillations, during the time interval $200<t<400$; later, it escapes because of the radiation loss.

another (pre-specified) position [38]. Apparently, this issue is important for applications, since it would demonstrate a possibility of driving the dark solitons (and other coherent structures, more generally) in the BEC by means of OL potentials. Note that it has been recently shown that an attractive steadily moving localized impurity may drag a stationary DS [10], but here we aim to consider the more general problem of the targeted transfer of a dark soliton by an OL.

Generally speaking, robust targeted transfer of solitons (in continua or lattices) may be implemented in dissipative systems by means of an external time-dependent force [39]. In the present case, as we aim to transfer the DS by a moving OL, we consider an OL potential of the following form:

$$
V_{\mathrm{OL}}(x, t)=V_{0} \cos ^{2}[k(x-y(t))],
$$

where the time-varying position $y(t)$, which will play the role of the "driver", is taken as

$$
y(t)=\eta_{\mathrm{i}}+\frac{1}{2}\left(\eta_{\mathrm{f}}-\eta_{\mathrm{i}}\right)\left[1+\tanh \left(\frac{t-t_{0}}{T}\right)\right] .
$$

In Eq. (19), $\eta_{\mathrm{i}}$ and $\eta_{\mathrm{f}}$ are the initial and final positions of the DS, while $T$ and $t_{0}$ are constants controlling, respectively, the duration and the beginning of the transfer. Note that the maximum of the transfer velocity $v_{\max } \equiv|\max (\mathrm{d} y / \mathrm{d} t)|=\left|\eta_{\mathrm{i}}-\eta_{\mathrm{f}}\right| / 2 T$, should be sufficiently small to ensure adiabaticity.

Simulations of the GP equation (1) with the OL potential as in Eq. (18) demonstrate the possibility of the DS transfer. As shown in Fig. 8, the soliton, initially placed at $\eta_{\mathrm{i}}=-3 \pi / 2$, is safely delivered to the new location, $\eta_{\mathrm{f}}=\pi / 2$ (the parameters were chosen to be $\Omega=0.025, V_{0}=0.07, k=1-$ the same as in Fig. $5-$ and $t_{0}=100, T=60$ ). We stress that, although the DS oscillates in the OL well, where it was initially captured, it remains in the trapped state, and is dragged by the moving OL very robustly. When the OL ceases to move, the DS performs small-amplitude oscillations in the well located at the final destination, $x=\eta_{\mathrm{i}}$. The soliton remains well-trapped there for a considerable time (approximately in the interval $200<t<400$ ). However, similar to what was shown in detail above, the radiative loss eventually leads to escape of the DS. This is seen in Fig. 8 for $t>400$. 
It should be stressed here that the example shown in Fig. 8 corresponds to a typical situation of what we have observed in targeted transfer numerical experiments. Direct simulations have shown that the proposed process is very robust for $v_{\max }<0.08$. Thus, we use Eq. (19) as a smooth interpolant between initial and final positions with a smoothly varying speed. Other particular forms of the dragging yield results very similar to those obtained with Eq. (19).

\section{Conclusions}

In this work, we have investigated the dark soliton (DS) dynamics in spatially inhomogeneous media. The analysis was based on a one-dimensional nonlinear Schrödinger equation incorporating external (spatially varying) potentials, which was treated both analytically and numerically. The considered form of the inhomogeneity suggests that the analysis and the results are relevant to cigar-shaped repulsive BECs confined in a harmonic magnetic trap (MT) together with an optical lattice (OL). Emphasis was placed on the long-time description of the DS dynamics, which is a quite relevant issue from the point of view of the theory of nonlinear waves and solitons; nevertheless, from the point of view of the physical situation encountered in relevant BEC experiments, dissipative mechanisms (thermal instabilities and quantum depletion of DSs) may reduce the soliton lifetime.

We have separately investigated the cases where the OL period was long or short, as compared to the healing length, which sets the spatial width of the DS. Both cases were treated analytically, the former by means of the adiabatic perturbation theory for DSs, and the latter by means of a multiple-scales perturbation technique. It has been found that, for both descriptions, the soliton performs oscillations in the condensate, whose frequencies and amplitudes were estimated analytically and calculated numerically with good agreement. Radiation effects, which become important in the case of strong OLs, were also investigated. It was found that, by shedding sound waves (small amplitude radiation waves), the soliton becomes more mobile, which results in the increase of the amplitude and decrease of the frequency of the oscillations.

We have also examined the case where the OL period and the width of the dark soliton are comparable. We have found that if the DS is initially placed quite close to the bottom of a well of the OL potential, it remains there for a rather long time; eventually, however, it escapes due to the radiation-loss mechanism, and then performs large-amplitude oscillations in the condensate. Furthermore, if the MT is weak enough, the soliton eventually decays. Based on the fact that the soliton may remain stationary for a relatively long time, we have proposed an experimentally realizable scheme to capture and drag the soliton by a slowly moving OL, bringing it to a pre-selected final destination. This transfer mechanism is robust, as long as the OL speed is small enough to ensure adiabaticity. Thus, time-dependent OLs may be used to manipulate the DS motion in the condensate. Work is in progress to demonstrate that a similar technique may also be used to control the motion of nonlinear excitations also in higher-dimensional BECs.

\section{Acknowledgements}

This work was supported by the "A.S. Onasis" Public Benefit Foundation (GT), the Special Research Account of the University of Athens, under grant no. 70/4/5844 (GT, DJF), a UMass FRG, the Eppley Foundation for Research and NSF-DMS-0204585 (PGK), the Binational (US-Israel) Science Foundation, 
under grant no. 1999459, and the European Office of Aerospace Research and Development of the US Air Force (BAM), and San Diego State University Foundation (RCG). Constructive discussions with N.P. Proukakis are kindly acknowledged.

\section{References}

[1] Ph. Emplit, et al., Opt. Commun. 62 (1987) 374; D. Krökel, N.J. Halas, G. Giuliani, D. Grischkowsky, Phys. Rev. Lett. 60 (1988) 29.

[2] D.R. Andersen, et al., Opt. Lett. 15 (1990) 783; G.A. Swartzlander Jr., D.R. Andersen, J.J. Regan, H. Yin, A.E. Kaplan, Phys. Rev. Lett. 66 (1991) 1583.

[3] Yu.S. Kivshar, G.P. Agrawal, Optical Solitons: From Fibers to Photonic Crystals, Academic Press, 2003.

[4] B. Denardo, W. Wright, S. Putterman, Phys. Rev. Lett. 64 (1990) 1518.

[5] B. Denardo, B. Galvin, A. Greenfield, A. Larraza, S. Putterman, W. Wright, Phys. Rev. Lett. 68 (1992) 1730.

[6] M. Chen, M.A. Tsankov, J.M. Nash, C.E. Patton, Phys. Rev. Lett. 70 (1993) 1707.

[7] F. Dalfovo, S. Giorgini, L.P. Pitaevskii, S. Stringari, Rev. Mod. Phys. 71 (1999) 463.

[8] S. Burger, K. Bongs, S. Dettmer, W. Ertmer, K. Sengstock, A. Sanpera, G.V. Shlyapnikov, M. Lewenstein, Phys. Rev. Lett. 83 (1999) 5198;

J. Denschlag, et al., Science 287 (2000) 97;

B.P. Anderson, et al., Phys. Rev. Lett. 86 (2001) 2926;

Z. Dutton, et al., Science 293 (2001) 663.

[9] W.P. Reinhardt, C.W. Clark, J. Phys. B 30 L785 (1997);

Th. Busch, J.R. Anglin, Phys. Rev. Lett. 84 (2000) 2298;

G. Huang, J. Szeftel, S. Zhu, Phys. Rev. A 65 (2002) 053605;

V.A. Brazhnyi, V.V. Konotop, Phys. Rev. A 68 (2003) 043613.

[10] D.J. Frantzeskakis, G. Theocharis, F.K. Diakonos, P. Schmelcher, Yu.S. Kivshar, Phys. Rev. A 66 (2002) 053608.

[11] P.O. Fedichev, A.E. Muryshev, G.V. Shlyapnikov, Phys. Rev. A 60 (1999) 3220; A. Muryshev, G.V. Shlyapnikov, W. Ertmer, K. Sengstock, M. Lewenstein, Phys. Rev. Lett. 89 (2002) 110401.

[12] D.L. Feder, M.S. Pindzola, L.A. Collins, B.I. Schneider, C.W. Clark, Phys. Rev. A 62 (2000) 053606;

J. Brand, W.P. Reinhardt, Phys. Rev. A 65 (2002) 043612.

[13] G. Theocharis, D.J. Frantzeskakis, P.G. Kevrekidis, B.A. Malomed, Yu.S. Kivshar, Phys. Rev. Lett. 90 (2003) 120403.

[14] N.G. Parker, N.P. Proukakis, M. Leadbeater, C.S. Adams, Phys. Rev. Lett. 90 (2003) 220401;

N.G. Parker, N.P. Proukakis, M. Leadbeater, C.S. Adams, J. Phys. B 36 (2003) 2891;

N.G. Parker, N.P. Proukakis, C.F. Barenghi, C.S. Adams, J. Opt. B: Quantum Semiclass. Opt. 6 (2004) S380;

N.P. Proukakis, N.G. Parker, D.J. Frantzeskakis, C.S. Adams, J. Opt. B 6 (2004) S380.

[15] J. Dziarmaga, K. Sacha, Phys. Rev. A 66 (2002) 043620;

J. Dziarmaga, Z.P. karkuszewski, K. Sacha, J. Phys. B 36 (2003) 1217;

C.K. Law, Phys. Rev. A 68 (2003) 015602.

[16] B.A. Anderson, M.A. Kasevich, Science 282 (1998) 1686.

[17] C. Orzel, A.K. Tuchman, M.L. Fenselau, M. Yasuda, M.A. Kasevich, Science 291 (2001) 2386.

[18] O. Morsch, J.H. Müller, M. Cristiani, D. Ciampini, E. Arimondo, Phys. Rev. Lett. 87 (2001) 140402.

[19] F.S. Cataliotti, S. Burger, C. Fort, P. Maddaloni, F. Minardi, A. Trombettoni, A. Smerzi, M. Inguscio, Science 293 (2001) 843.

[20] M. Greiner, I. Bloch, O. Mandel, T.W. Hänsch, T. Esslinger, Phys. Rev. Lett. 87 (2001) 160405.

[21] M. Greiner, O. Mandel, T. Esslinger, T.W. Hänsch, I. Bloch, Nature (London) 415 (2002) 39.

[22] D.I. Choi, Q. Niu, Phys. Rev. Lett. 82 (1999) 2022.

[23] A. Smerzi, A. Trombettoni, P.G. Kevrekidis, A.R. Bishop, Phys. Rev. Lett. 89 (2002) 170402;

F.S. Cataliotti, L. Falani, F. Ferlaino, C. Fort, P. Maddaloni, M. Inguscio, A. Smerzi, A. Trombettoni, P.G. Kevrekidis, A.R. Bishop, N. J. Phys. 5 (2003) 71.

[24] N.G. Parker, N.P. Proukakis, C.F. Barenghi, C.S. Adams, J. Phys. B: At. Mol. Opt. Phys. 37 S175 (2004). 
[25] P.J.Y. Louis, E.A. Ostrovskaya, Yu.S. Kivshar, J. Opt. B: Quantum Semiclass. Opt. 6 S309 (2004).

[26] P.G. Kevrekidis, R. Carretero-González, G. Theocharis, D.J. Frantzeskakis, B.A. Malomed, Phys. Rev. A 68 (2003) 035602.

[27] A.R. Bishop, D.K. Campbell, St. Pnevmatikos (Eds.), Disorder and Nonlinearity, Springer Proceedings in Physics vol. 39, Springer-Verlag, Berlin, 1989;

F.Kh. Abdullaev, A.R. Bishop, St. Pnevmatikos (Eds.), Nonlinearity with Disorder, Springer Proceedings in Physics, vol. 67, Springer-Verlag, Berlin, 1992;

Yu.S. Kivshar, B.A. Malomed, Rev. Mod. Phys. 61 (1989) 763.

[28] R. Scharf, A.R. Bishop, Phys. Rev. A 46 (1992) R2973;

R. Scharf, A.R. Bishop, Phys. Rev. A 47 (1993) 1375;

R. Scharf, Chaos, Solitons Fractals 5 (1995) 2527.

[29] P.A. Ruprecht, M.J. Holland, K. Burnett, M. Edwards, Phys. Rev. A 51 (1995) 4704.

[30] V.M. Pérez-García, H. Michinel, H. Herrero, Phys. Rev. A 57 (1998) 3837;

L. Salasnich, A. Parola, L. Reatto, Phys. Rev. A 65 (2002) 043614;

Y.B. Band, I. Towers, B.A. Malomed, Phys. Rev. A 67 (2003) 023602.

[31] O. Morsch, E. Arimondo, T. Dauxois, S. Ruffo, E. Arimondo, M. Wilkens (Eds.), Dynamics and Thermodynamics of Systems with Long-Range Interactions, Springer, Berlin, 2002, pp. 312-331.

[32] V.E. Zakharov, A.B. Shabat, Zh. Eksp. Teor. Fiz. 64 (1973) 1627;

V.E. Zakharov, A.B. Shabat, Sov. Phys. JETP 37 (1973) 823.

[33] Yu.S. Kivshar, X. Yang, Phys. Rev. E 49 (1994) 1657.

[34] Yu.S. Kivshar, S.K. Turitsyn, Phys. Rev. E 49 (1994) R2536;

Yu.S. Kivshar, K.H. Spatschek, Chaos, Solitons Fractals 5 (1995) 2551;

F.Kh. Abdullaev, R. Galimzyanov, J. Phys. B: At. Mol. Opt. Phys. 36 (2003) 1099.

[35] T.R. Taha, M.J. Ablowitz, J. Comp. Phys. 55 (1984) 203;

V.M. Pérez-García, X.Y. Liu, Appl. Math. Comput. 144 (2003) 215.

[36] M. Krämer, L. Pitaevskii, S. Stringari, Phys. Rev. Lett. 88 (2002) 180404.

[37] D.E. Pelinovsky, Yu.S. Kivshar, V.V. Afanasjev, Phys. Rev. E 54 (1996) 2015;

D.E. Pelinovsky, D.J. Frantzeskakis, P.G. Kevrekidis, cond-mat/0503657, Phys. Rev. E, in press.

[38] G. Theocharis, D.J. Frantzeskakis, R. Carretero-González, P.G. Kevrekidis, B.A. Malomed, Phys. Rev. E 71 (2005) 017602.

[39] H.E. Nistazakis, P.G. Kevrekidis, B.A. Malomed, D.J. Frantzeskakis, A.R. Bishop, Phys. Rev. E 66 (2002) 015601R. 